\title{
Spotlight on adalimumab in the treatment of active moderate-to-severe hidradenitis suppurativa
}

This article was published in the following Dove Press journal:

Clinical, Cosmetic and Investigational Dermatology

19 October 2016

Number of times this article has been viewed

\section{Christina Fotiadou \\ Efstratios Vakirlis \\ Dimitrios loannides \\ First Department of Dermatology- Venereology, Aristotle University Medical School, Thessaloniki, Greece}

\begin{abstract}
Hidradenitis suppurativa (HS) is a chronic, recurrent, inflammatory skin disease that affects the hair follicles of the aprocrine gland-bearing anatomical areas of the body. It is characterized by deep painful nodules and abscesses that rupture and contribute to the formation of sinus tracks and scarring. The management of HS is based on the assessment of disease severity and a combination of medical and surgical treatment according to the European Guidelines. Adalimumab, a recombinant, fully humanized, anti-tumor necrosis factor alpha (anti-TNF- $\alpha$ ) monoclonal antibody, is the only officially approved treatment for the management of moderateto-severe HS. Case reports, concerning 42 patients who received adalimumab for severe HS (with the standard dose regimen for psoriasis), reported a cumulative response rate of $58 \%$ ( $\geq 50 \%$ in 23 patients) with a relapse rate of $71 \%$ (10 out of 14 patients). The most recent and most well-powered phase III, randomized placebo-controlled trials for the evaluation of the efficacy and safety of adalimumab in treatment of moderate-to-severe HS (PIONEER studies I and II) showed that the Hidradenitis Suppurativa Clinical Response (HiSCR) rate at week 12 was significantly higher for patients randomized to adalimumab compared to placebo. Adverse events were comparable to placebo. In conclusion, adalimumab, to date, holds the most robust data regarding treatment efficacy in HS. Larger, registry-based studies are needed to further establish the efficacy and safety profile of this anti-TNF- $\alpha$ agent in HS.
\end{abstract}

Keywords: hidradenitis suppurativa, adalimumab, treatment

\section{Introduction}

Hidradenitis suppurativa (HS) is a chronic, recurrent, inflammatory skin disease that affects the hair follicles of the aprocrine gland-bearing anatomical areas of the body. ${ }^{1}$ It is characterized by deep painful nodules and abscesses that rupture and contribute to the formation of sinus tracks and scarring. ${ }^{2}$ The axillae, inguinal, anogenital regions and mammary, and inframammary areas are the most commonly affected parts of the body. ${ }^{3}$ The diagnosis of the disease is based on the following findings: history of recurrent, painful, suppurating lesions in intertrigenous body areas for two or more times in 6 months. $^{2}$

The exact prevalence of the disease is uncertain, though, it is believed that it ranges between $1 \%$ and $4 \% .{ }^{4}$ Its estimated incidence - according to a study by Vazquez et al - reaches 6.0 cases per 100,000 person years. ${ }^{5-7}$ HS most frequently occurs in young adults during the second and third decade of their life. ${ }^{8,9}$ There is a characteristic female predominance (female to male ratio 3:1), although males tend to suffer from more severe symptoms of the disease. ${ }^{10}$
Correspondence: Dimitrios loannides First Department of DermatologyVenereology, Aristotle University Medical School, 124 Delfon street, 52440 ,

Thessaloniki, Greece Email dem@auth.gr 
Follicular occlusion is now considered the main pathogenetic event in HS. ${ }^{11}$ Evidently, an imbalance of the immune system which precedes and/or follows follicular occlusion - in genetically predisposed individuals - also plays an important role in course of the disease. ${ }^{3,12-14}$ A genetic background, expressed with an autosomal dominant inheritance pattern, is present in about $40 \%$ of patients suffering from HS. ${ }^{15}$ Certain trigger factors such as smoking ( $70 \%-88.9 \%$ of cases), obesity ( $50 \%$ of cases), mechanical friction and to a lesser extent, hormones, drugs, and depilation seem to contribute to the manifestation of this entity. ${ }^{16-20} \mathrm{~A}$ matter of controversy among authors is the possibility that the alteration of microbial flora and the formation of bacterial biofilms could act as a predisposing factor for HS patients. ${ }^{21-23}$ HS has been associated with several comorbid autoinflammatory disorders (inflammatory bowel disease and spondyloarthropathy) as well as pyoderma gangrenosum and epithelial tumors (squamous cell carcinomas). ${ }^{1,5,24-26}$

HS patients experience a negative impact on physical, social, and emotional aspects of their life. ${ }^{27}$ As a consequence, patients have a poor quality of life that is assessed using the Dermatology Life Quality Index (DLQI), whose value ranges between 8.4 and 12.7 in several studies. ${ }^{28-30}$

\section{Advances in the management of HS}

The strategic management of HS is based on the assessment of disease severity and a combination of medical and surgical treatment according to the European Guidelines. ${ }^{2,31}$ All patients with HS could benefit from adjuvant therapy which may include pain management, treatment of super-infections, as well as weight loss and smoking cessation. ${ }^{2,31}$

In terms of surgical intervention, procedures such as deroofing, laser, local excision, or even wide excision could be implemented depending on the type and extent of abscesses and the severity of scaring in each individual case., ${ }^{2,31}$

The management of the inflammatory component of the HS is based on medical treatment. For mild disease (1-2 small abscesses or inflammatory nodules), topical clindamycin $1 \%$ twice daily may be helpful. If the patient does not respond or has moderate-to-severe disease, the administration of tetracycline $500 \mathrm{mg}$ po (orally) twice daily for 4 months or even the combination of clindamycin $300 \mathrm{mg}$ po twice daily along with rifampicin $600 \mathrm{mg}$ once daily for 10 weeks should be considered. ${ }^{2,31}$ The next treatment choice in the occasion of no improvement is adalimumab $160 \mathrm{mg}$ at week $0,80 \mathrm{mg}$ at week 2 , and then $40 \mathrm{mg}$ subcutaneously (sc) every week. ${ }^{31}$ If this treatment is effective, it should be maintained as long as the lesions are present. ${ }^{31}$ In the case of no response, second-line (infliximab, acitretin) and third-line (cyclosporine, dapsone, hormones, isotretinoin, alitretinoin, colchicines) treatments should be tried. ${ }^{2,31}$

\section{Rationale for the use of adalimumab in the treatment of HS}

Adalimumab is the only officially approved treatment for the management of moderate-to-severe HS by both the European Medicines Agency (EMA, June 2015) and the US Food and Drug Administration (FDA, September 2015) with a recommended dose of $160 \mathrm{mg} \mathrm{sc}$ at week $0,80 \mathrm{mg} \mathrm{sc}$ at week 2, and then $40 \mathrm{mg}$ every week starting from week 4. It is a recombinant, fully humanized, anti-tumor necrosis factor alpha (anti-TNF- $\alpha$ ) monoclonal antibody (IgG1) that has high affinity and specificity for TNF- $\alpha$. Adalimumab binds both soluble and membrane-bound forms of TNF- $\alpha$ and inhibits its activities by blocking its interaction with $\mathrm{p} 55$ and $\mathrm{p} 75$ cell surface TNF- $\alpha$ receptors. ${ }^{32}$

Preliminary, serendipitous evidence that anti-TNF- $\alpha$ agents could be effective in the management of HS came from the discipline of gastroenterology and was first reported in patients with associated Crohn's disease.$^{33}$ These patients were treated with anti-TNF- $\alpha$ agents for their Crohn's disease and simultaneously exhibited clear improvement in their HS. ${ }^{33,34}$ Based on these observations, several studies have been conducted in order to unravel a possible role of TNF- $\alpha$ and other proinflammatory cytokines in the pathogenesis of HS. ${ }^{35-37}$ Moreover, case reports, series, and later clinical trials have focused on the efficacy of adalimumab and other TNF- $\alpha$ inhibitors in the treatment of HS.

Indeed, in an in vivo study by Matusiak et al, the serum concentration of TNF- $\alpha$ in patients with HS was significantly higher compared with that of healthy controls, although it was not correlated with disease severity. ${ }^{35}$ In another study by van der Zee et al, the levels of TNF- $\alpha$ along with interleukin $1 \beta$ (IL-1 $1 \beta$ ) and IL-10 were elevated in lesional and perilesional skin of patients with HS compared with healthy controls and even psoriatic skin. ${ }^{36}$ These results demonstrated the strongly inflammatory nature of HS and showed that inflammation extends beyond the visibly inflamed border. ${ }^{36}$ The same author in another in situ and ex vivo study, which was part of larger placebo-controlled double-blind phase IIb trial on the efficacy and safety of adalimumab in patients with moderate-to-severe HS, showed that adalimumab treatment inhibits important cytokines (IL-1 $\beta$ ) and inflammatory cell 
numbers (CD11 c+ dendritic cells) in lesional skin. ${ }^{37}$ Also, in this study, it was evident that lack of clinical improvement highly correlated with the lack of alterations in the leucocyte subset scores and cytokine levels. ${ }^{37}$

\section{Real life evidence about the use of adalimumab in HS - early case series and open-label studies}

The first data about the efficacy of adalimumab on HS came from several case series in the majority of which the drug was administered with the standard dose regimen of psoriasis (80 $\mathrm{mg} \mathrm{sc}$ at week $0,40 \mathrm{mg} \mathrm{sc}$ at week 1 , and then $40 \mathrm{mg} \mathrm{sc}$ every other week). Unfortunately, in each study a different tool for the evaluation of improvement was used and this did not allow for direct comparisons between them.

In general, case reports concerning 42 patients who received adalimumab for severe HS reported a cumulative response rate of $58 \%$ ( $\geq 50 \%$ in 23 patients) with a relapse rate of $71 \%$ (10 out of 14 patients). ${ }^{2,38-41}$ In the majority of patients, the drug was well tolerated. ${ }^{2}$

In a study by Arenbergerova et al, eight patients with severe HS were treated for 1 year with adalimumab in a standard psoriasis regimen and were subsequently followed for 1 year. ${ }^{40}$ All patients improved within 4-6 weeks. Three of them demonstrated long-lasting improvement, while five showed recurrences after a mean disease-free period of 9.5 months. ${ }^{40}$ Blanco et al used adalimumab in six patients with the previously mentioned dose regimen. All of the patients exhibited significant improvements in DLQI and in the number of affected areas, nodules, and fistulas. These results were maintained for a mean follow-up period of 21.5 months. ${ }^{41}$

In two open-label prospective studies, adalimumab was administered in doses higher than the approved regimen for psoriasis. Amano et al treated 10 HS patients with adalimumab for 12 weeks at doses of $160 \mathrm{mg}$ sc at week $0,80 \mathrm{mg}$ sc at week 1 , and the $40 \mathrm{mg}$ sc every other week. ${ }^{42}$ Clinically significant improvement was not observed in any of the six patients who completed the study. ${ }^{42}$ Sotiriou et al in another open, prospective study with 15 patients suffering from moderate-to-severe HS administered adalimumab with the following dose: $80 \mathrm{mg}$ sc at week 0 and then $40 \mathrm{mg}$ sc every week for 24 weeks. Significant reduction in Sartorius score was obtained at week 24 with a marked improvement during the first month. ${ }^{43}$ Mean time to relapse was 11 weeks after treatment cessation, but even at the final visit, Sartorius score was lower than at baseline. ${ }^{43}$

\section{Randomized controlled trials (RCTs) for the evaluation of the efficacy and safety of adalimumab in the treatment of HS Early RCTs}

Miller et al conducted a prospective, double-blind, placebocontrolled clinical trial which included 21 patients who suffered from severe HS (Hurley stage II and III) for at least 6 months. ${ }^{44}$ Fifteen patients received adalimumab ( $80 \mathrm{mg}$ sc at baseline followed by $40 \mathrm{mg}$ every other week) for 12 weeks, while seven patients received placebo (1:2 placebo/active).$^{44} \mathrm{~A}$ significant reduction in Sartorius score was seen after 6 weeks and an almost significant reduction after 12 weeks of active treatment when compared with placebo $(P=0.024$ and $P=0.07$, respectively). ${ }^{44}$ Adalimumab was well tolerated, in general, but the active group experienced an almost significantly greater number of adverse events (mostly mild infections). ${ }^{44}$

Kimbal et al conducted an even larger double-blind placebo-controlled randomized trial that included 154 patients with moderate-to-severe HS who were unresponsive or intolerant to oral antibiotics. ${ }^{45}$ Patients were randomized to receive adalimumab (1:1:1) either $40 \mathrm{mg}$ sc once weekly or $40 \mathrm{mg} \mathrm{sc}$ every other week or placebo for 16 weeks (period 1). ${ }^{45}$ Then all patients received adalimumab, $40 \mathrm{mg}$ every other week, at the beginning of period 2, but switched to weekly dosing if the response was suboptimal (HS-PGA score of moderate or worse) at weeks 28 or $31 .^{45}$ At week 16, clinical responses (i.e. an HS-PGA score of clear, minimal, or mild with at least a 2-grade improvement relative to baseline) were achieved by $17.6 \%$ of weekly patients, $9.6 \%$ of every-other-week patients, and $3.9 \%$ of placebo patients. ${ }^{45}$ A decrease in response was observed after the switch from weekly to every-other-week dosing in period $2 .{ }^{45}$ Headache and injection site reactions were the most common adverse events, while the serious adverse event rates were $3.9 \%$, $5.8 \%$, and $7.8 \%$ for placebo, every-other-week, and weekly patients, respectively. ${ }^{45}$

\section{PIONEER study}

The most recent and most well-powered phase III, randomized placebo-controlled trial for the evaluation of the efficacy and safety of adalimumab in treatment of moderate-to-severe HS is the PIONEER study ( I and II). ${ }^{46,47}$

In this study, patients who had an established diagnosis (duration $\geq 1$ year) of moderate-to-severe HS (lesions in $\geq 2$ distinct body areas, one of which must be Hurley II or III) 
were randomized $(1: 1)$ to receive either placebo or adalimumab $40 \mathrm{mg}$ weekly for 12 weeks (period A). Specifically, the adalimumab group received $160 \mathrm{mg}$ at week 0 , followed by $80 \mathrm{mg}$ at week 2 and $40 \mathrm{mg}$ weekly from week 4 through week 12. After the initial period A, they were re-randomized to receive adalimumab either $40 \mathrm{mg}$ weekly or $40 \mathrm{mg}$ every other week versus placebo. Those patients who were in the placebo group during period A were randomized to receive either $40 \mathrm{mg}$ weekly (PIONEER I) or placebo (PIONEER II).

In total, 633 patients participated in these two studies (PIONEER I: 307, PIONEER II: 321). The primary efficacy end point, in both studies, was the proportion of patients achieving a Hidradenitis Suppurativa Clinical Response (HiSCR), defined as $>50 \%$ reduction from baseline in total abscess and inflammatory nodule count, and no increase in abscess and draining fistula count. Secondary end points were the proportion of patients who achieved $\geq 30 \%$ reduction from baseline (partial response) and the change in modified Sartorius score from baseline to week 12, among others.

In PIONEER I (period A) study, a significantly higher proportion of patients randomized to adalimumab achieved the primary efficacy end point HiSCR at week 12: adalimumab $(64 / 153,41.8 \%)$ versus placebo (40/154, 26.0\%; $P=0.003)$. Adverse events were comparable to placebo and consistent with the adalimumab safety profile; no new risks were identified. In PIONEER II (period B) study, HiSCR rate at week 12 was significantly higher for patients randomized to adalimumab $(96 / 163,58.9 \%)$ versus placebo $(45 / 163$, $27.6 \% ; P<0.001)$. Statistically significant differences in the treatment were observed for all secondary end points, with more emphasis to the marked reduction in skin pain. DLQI was greatly improved in the adalimumab-treated patients and $50 \%$ of them reached the minimal clinically important difference, thus achieving a clear improvement in the quality of life. ${ }^{48}$ The safety profile for patients in both treatment groups was comparable. It must be pointed out that in both studies the improvement observed in the adalimumab group was evident as early as week 2 , when compared to placebo.

\section{Comparison with other biologic agents}

Infliximab is a chimeric mouse/human monoclonal antibody against both the membrane-bound and soluble TNF- $\alpha$. It has the longest "tradition" in the treatment of HS since the first observations about a possible role of anti-TNF- $\alpha$ agents in the management of HS were made in patients with inflammatory bowel disease who suffered also from HS and were treated with infliximab. ${ }^{33,34}$ Ten cohort studies with at least four and no more than 11 patients in each study (around 73 patients in total) have been published regarding the use of infliximab in moderate-to-severe HS. ${ }^{2,3,49}$ Almost all of these studies found that some of the patients improved with treatment. However, it seems that the best results were achieved with a more intensified regimen of $5 \mathrm{mg} / \mathrm{kg}$ at weeks $0,2,6$ and monthly thereafter, compared with the infliximab treatment scheme for psoriasis. ${ }^{50} \mathrm{~A}$ recurrence rate of about $43 \%$ was observed after cessation of treatment in these case reports. ${ }^{2}$ The only randomized placebo-controlled trial on the use of infliximab in the treatment of HS was the one performed by Grant et al. ${ }^{51}$ In this 8-week study, 33 patients with moderateto-severe HS were treated with infliximab $5 \mathrm{mg} / \mathrm{kg}$ at weeks 0,2 , and 6 or placebo. After the eighth week, there was a crossover and patients from the placebo group could take infliximab with the previously mentioned regimen. The outcome measure used in this study was HS Severity Index (HSSI). At week 8, 25\% of patients had at least 50\% reduction of HSSI from baseline, but this did not reach statistical significance versus placebo (primary efficacy outcome was not achieved). ${ }^{51}$ However, $20 \%-60 \%$ of patients, at week 8 , reached a $25 \%-50 \%$ improvement in HSSI which was statistically significant compared with placebo. ${ }^{51}$ DLQI also showed considerable improvement. ${ }^{51}$ Recurrences occurred after discontinuation of treatment. In a retrospective comparative study with $20 \mathrm{HS}$ patients (1:1), infliximab achieved a significantly greater improvement in mean Sartorius score $(56 \%)$ compared with adalimumab $(34 \%) .{ }^{50}$ Infliximab was administered at $5 \mathrm{mg} / \mathrm{kg}$ intravenously at weeks 0,2 , and 6 , while adalimumab was given at $40 \mathrm{mg}$ sc every other week, which is not the recommended dose for HS. ${ }^{50} \mathrm{~A}$ very recent retrospective national cohort study, which included 67 patients who received anti-TNF- $\alpha$ agents for HS, concluded that adalimumab seems to be more effective than infliximab and etanercept in the treatment of severe HS. ${ }^{52}$

Etanercept is a fusion recombinant protein, which fuses with the TNF receptor and interferes with TNF- $\alpha$ binding. According to several case reports, it has been administrated with a dose regimen of $25 \mathrm{mg}$ sc twice weekly, in about 34 patients with encouraging outcomes. ${ }^{2,53-54}$ However, in the only randomized placebo-controlled trial on the use of etanercept in HS ( $\mathrm{n}=20$ patients), the dose regimen was $50 \mathrm{mg}$ sc twice weekly for 3 months and there was no significant difference compared with placebo. ${ }^{55}$

Ustekinumab (a human monoclonal antibody against the p40 subunit of IL-12/IL-23) in treatment of HS has been 
described in one case report with three patients. ${ }^{56}$ The dose regimen used was $45 \mathrm{mg} \mathrm{sc}$ at weeks 0,4 , and 16 . One of the three patients (33\%) exhibited an improvement of $\geq 50 \%$. ${ }^{56}$ However, it is too early to decisively conclude about the effectiveness of this biologic agent in psoriasis.

Anakinra is a recombinant form of the human IL-1 receptor antagonist that inhibits the biologic activity of IL- $1 \alpha$ and IL-1 $\beta$. It has been tried in several cases of severe HS. Initial sporadic case reports have produced contradictory outcomes, while an open-label study by Leslie et al has shown a satisfactory response (i.e. decrease of Sartorius score) in five out of six patients. ${ }^{57-60}$ Recently, Tzanetakou et al conducted a double-blind, randomized, placebo-controlled clinical trial with a very promising outcome. ${ }^{61}$ It included 10 patients who received once-daily subcutaneous injection of anakinra 100 mg for 12 weeks and 10 controls. An HiSCR was achieved by $78 \%$ of patients in the anakinra group and by only $20 \%$ of patients in the control group. However, larger studies are needed in order to confirm these results.

\section{Conclusion}

In the era of evidence-based medicine and the new understanding of the inflammatory pathways that characterize HS, adalimumab, to date, holds the most robust data regarding efficacy in the treatment of the disease. Apparently, adalimumab can contribute to the optimal control of the inflammatory burden of the disease and hopefully in a second level could prevent scarring. However, future larger registry-based studies are needed to further establish the efficacy and safety profile of this anti-TNF- $\alpha$ agent in HS.

\section{Disclosure}

The authors report no conflicts of interest in this work.

\section{References}

1. Fimmel S, Zoumboulis CC. Comordidities of hidradenitis suppurativa (acne inversa). Dermatoendocrinol. 2010;2:9-16.

2. Zoumboulis CC, Dessai N, Emtestam L, et al. European S1 guideline for the treatment of hidradenitis supppurativa/acne inversa. J Eur Acad Dermatol Venereol. 2015;29:619-644.

3. Martorell A, Garcia-Martinez FJ, Jimenez-Gallo D, et al. An update on hidradenitis suppurativa (part I): epidemiology, clinical aspects and definition of disease severity. Actas Dermosifiliogr. 2015;106:703-715.

4. Jemec GB, Kimbal AB. Epidemiology and scope of the problem. J Am Acad Dermatol. 2015;73:S4-S7.

5. Revuz JE, Canoui-Poitrine F, Wolkstein R, et al. Prevalence and factors associated with hidradenitis suppurativa: results from two case controls studies. J Am Acad Dermatol. 2008;59:596-601.

6. Jemec GB, Heidenheim M, Nielsen NH. The prevalence of hidradenitis suppurativa and its potential precursor lesions. J Am Acad Dermatol. 1996;35:191-194.
7. Vazquez BG, Alikhan A, Weaver AL, Wetter DA, Davis MD. Incidence of hidradenitis suppurativa and associated factors: a population-based study of Olmsted county, Minnesota. J Invest Dermatol. 2013;133: 97-103.

8. Cannoui-Poitrine F, Revuz JE, Wolkenstein P et al. Clinical charecterisitcs of a series of 302 French patients with hidradenitis suppurativa, with an analysis of factors associated with disease severity. $J$ Am Acad Dermatol. 2009:61:51-57.

9. Michelletti RG. Natural history, presentation and diagnosis of hidradenitis suppurativa. Semin Cutan Med Surg. 2014;33(Suppl 3):S51-S53.

10. Matusiak L, Bienek A, Szepietowski JC. Hidradenitis Supurativa and associated factors: still unsolved problems. J Am Acad Dermatol. 2009;61:362-365.

11. Prens E, Deckers I. Pathophysiology of hidradenitis suppurativa: an update. J Am Acad Dermatol 2015;73:S8-S11.

12. Barlev D, Eisen DB, Alikhan A. Hidradenitis suppurativa a review with a focus on treatment data. Skin Therapy Lett. 2015;20:1-8.

13. Kelly G, Sweeney SM, Tobin AM, Kirby B. Hidradenitis suppurativa: the role of immune dysregulation. Int J Dermatol. 2014;53:1186-1196.

14. Dreno B, Khammari A, Brockard A, et al. Hidradenitis supurativa: the role of deficient cutaneous innate immunity. Arch Dermatol. 2012;148:182-186.

15. Wang B, Yang W, Wen W, et al. Gamma-secretase gene mutations in familial acne inversa. Science. 2010;330:1065.

16. Alikhan A, Lynch PJ, Eisen DB. Hidradenitis Suppurativa: a comprehensive review. J Am Acad Dermatol. 2009;60:539-561.

17. Deckers IE, van der Zee HH, Prens EP. Epidemiology of hidradenitis suppurativa: prevalence, pathogenesis, and factors associated with the development of HS. Curr Dermatol Rep. 2014;3:54-60.

18. Konig A, Lehmann C, Rompel R, Happle R. Cigarette smoking as a triggering factor of hidradenitis suppurativa. Dermatology. 1999;198:261-264.

19. Sartorius K, Emtestam L, Jemec GB, Lapins J. Objective scoring of hidradenitis suppurativa reflecting the role of tobacco smoking and obesity. Br J Dermatol. 2009;161:831-839.

20. Margesson LJ, Danby FW. Hidradenitis suppurativa . Best Pract Res Clin Obstet Gynaecol. 2014;28:1013-1027.

21. Michelleti RG. Hidradenitis suppurativa: current views on epidemiology, pathogenesis and pathophysiology. Semin Cutan Med Surg. 2014;33(Suppl 3):S48-S50.

22. Van der Zee HH, de Ruiter L, Boer J, van de Broecke DG, et al. Alterations in leucocyte subsets and histomorphology in normal-appearing perilesional skin and early and chronic hidradenitis suppurativa lesions. Br J Dermatol. 2012;166:98-106.

23. Kathju S, Lasko LA, Stoodley P. Considering hidradenitis suppurativa as a bacterial biofilm disease. FEMS Immunol Med Microbiol. 2012;65:385-389.

24. Scheinfield N. Diseases associated with hidradenitis suppurativa: Part 2 of a series on hidradenitis. Dermatol Online J. 2013;19:18558.

25. Van der Zee HH, van der Woude CJ, Florencia EF, Prens EP. Hidradenitis suppurativa and inflammatory bowel disease: are they associated? Results from a pilot study. Br J Dermatol. 2010;162:195-197.

26. Richette $P$, Molto A, Viguier M, et al. Hidradenitis suppurativa associated with spondyloarthritis-results from a multicenter national prospective study. J Rheumatol. 2014;53:1186-1196.

27. Gooderham M, Papp K. The psychological impact of hidradenitis suppurativa. J Am Acad Dermatol. 2015;73:S19-S22.

28. von de Werth JM, Jemec GB. Morbidity in patients with hidradenitis supurativa. Br J Dermatol. 2001;144:809-813.

29. Onderdijk AJ, van der Zee HH, Esmann S, et al. Depression in patients with hidradenitis suppurativa. J Eur Acad Dermatol Venereol. 2013;27:473-478.

30. Matusiak L, Bieniek K, Szepietowski JC. Psycophysical aspects of hidradenitis suppurativa. Acta Dermatol Venereol. 2010;90:264-268. 
31. Gulliver W, Zouboulis C, Prens E, Jemrc GB, Tzellos T. Evidence-based approach to the treatment of hidradenitis suppurativa/acne inversa, based on the European guidelines for hidradenitis suppurativa. Rev Endocr Metab Disord. Epub 2016 Feb 1.

32. European Medicines Agency. Humira ${ }^{\circledR}$ Summary of product characteristics [WWW document]; 2009 [updated 26 May 2016].Available from: http://www.ema.europa.eu/docs/en_GB/document_library/ EPAR_-_Product_Information/human/000481/WC500050870.pdf. Accessed June 13, 2016.

33. Hanauer SB, Feagan BG, Lichtenstein GR, et al. Maintenance infliximab for Crohn's disease: the ACCENT 1 randomised trial. Lancet. 2002;359:1542-1549.

34. Haslund P, Lee R, Jemec GB. Treatment of hidradenitis suppurativa with anti-tumor necrosis factor- $\alpha$ inhibitors. Acta Derm Venereol. 2009;89:595-600.

35. Matusiak L, Bieniek K, Szepietowski JC. Increased serum tumour necrosis factor-alpha in hidradenitis suppurativa patients: is there a basis for treatment with anti-tumour necrosis factor-alpha agents? Acta Derma Venereol (Stockh). 2009;89:601-603.

36. van der Zee HH, de Ruiter I, van de Broecke DG, Dik WA, Laman JD, Prens EP. Elevated levels of tumour necrosis factor (TNF)-a, interleukin (IL)-1 $\beta$ and IL-10 in hidradenitis suppurativa skin: a rationale for targeting TNF- $\alpha$ and IL-1 $\beta$. Br J Dermatol. 2011;164:1292-1298.

37. van der Zee HH, Laman JD, de Ruiter L, Dik WA, Prens EP. Adalimumab (antitumour necrosis factor- $\alpha$ ) treatment of hidradenitis suppurativa ameliorates skin inflammation: an in situ and ex vivo study. Br J Dermatol. 2012;166:298-305.

38. Sotiriou E, Apalla Z, Vakirlis E, Ioannides D. Efficacy of adalimumab in recalcitrant hidradenitis suppurativa. Eur J Dermatol. 2009;19:180-181.

39. Yamauchi PS, Mau N. Hidradenitis suppurativa managed with adalimumab. J Drugs Dermatol. 2009;8:181-183.

40. Arenbergerova M, Gkalpakiotis S, Arenberger P. Effective long-term control of refractory hidradenitis suppurativa with adalimumab after failure of conventional therapy. Int J Dermatol. 2010;49:1445-1449.

41. Blanco R, Martinez -Taboada VM, Villa L, et al. Long-term successful adalimumab therapy for hidradenitis suppurativa. Arch Dermatol. 2009; $145: 580-584$.

42. Amano M, Grant A, Kerdel FA. A prospective open-label clinical trial of adalimumab for the treatment of hidradenitis suppurativa. Int $J$ Dermatol. 2010; 49:950-955.

43. Sotiriou E, Goussi C, Lallas A, et al. A prospective open-label clinical trial of efficacy of the every week administration of adalimumab in the treatment of hidradenitis suppurativa. J Drugs Dermatol. 2012;11:s15-s20.

44. Miller I, Lynggaard CD, Zachariae C, Dufour DN, Jemec GB. A double blind placebo controlled randomized trial of adalimumab in the treatment of hidradenitis suppurativa. Br J Dermatol. 2011;165:391-398.

45. Kimbal A, Kerdel F, Adams D et al. Adalimumab for the treatment of moderate to severe hidradenitis suppurativa: a parallel randomized trial. Ann Intern Med. 2012;157:846-855.
46. Kimbal A, Zouboulis C, Armstrong A, et al. Safety and efficacy of adalimumab in patients with severe hidradenitis suppurativa: results from PIONEER I, a phase 3 randomized, placebo-controlled trial. $J$ Am Acad Dermatol. 2015;72:S1(AB60, P: 507).

47. Jemec GB, Gottlieb A, Giamarellos-Bourboulis E, Reguiai Z, Gu Y, Okun M. Safety and efficacy of adalimumab in patients with severe hidradenitis suppurativa: results from PIONEER II, a phase 3 randomized, placebo-controlled trial. JAm Acad Dermatol. 2015;72:S1(AB45, P: 631).

48. Armstrong A, Pinsky B, Sundaram M, Okun M, Bao Y. Adalimumab improves health related quality of life (HRQoL) in patients with moderate to severe hidradenitis suppurativa (HS): results from the first weeks of PIONEER II. Presented at the $23^{\text {rd }}$ Annual meeting of the American Academy of Dermatology; March 20-24, 2015; San Fransisco, California.

49. Lee R, Eisen D. Treatment of hidradenitis suppurativa with biologic medications. J Am Acad Dermatol. 2015;73:S82-8.

50. Mortiary B, Jiyad Z, Creamer D. Four-weekly infliximab in the treatment of severe hidradenitis suppurativa. Br J Dermatol. 2014;170:986-987.

51. Grant A, Gonzalez T, Montgomery MO, Cardenas V, Kerdel FA. Infliximab therapy for patients with moderate hidradenitis suppurativa: a randomized, double-blind, placebo controlled crossover trial. JAm Acad Dermatol. 2010;62:205-217.

52. Sbidian E, Hotz C, Seneschal J, et al. Antitumour necrosis factor- $\alpha$ for hidradenitis suppurativa: results from a national cohort study between 200 and 2003. Br J Dermatol. 2016;174:667-670.

53. Sotiriou E, Apalla Z, Ioannides D. Etanercept for the treatment of hidradenitis suppurativa. Acta Derm Venereol. 2009;89:82-83.

54. Giamarellos-Bourboulis EI, Pelekanou E, Antonopoulou A, et al. An open label phase II study on the safety and efficacy of etanercept for the therapy of hidradentis suppurativa. Br J Dermatol. 2008;158:567-572.

55. Adams DR, Yankura IA, Fogelberg AC, Anderson BE. Treatment of hidradenitis suppurativa with etanercept injection. Arch Dermatol. 2010;146:501-504.

56. Gulliver WP, Jemec GB, Baker KA. Experience with ustekinumab for the treatment of moderate to severe hidradenitis suppuratiav. J Eur Acad Dermatol Venereol. 2012;26:911-914.

57. Russo V, Alikhan A. Failure of Anakinra in a case of severe hidradenitis suppurativa. J Drugs Dermatol. 2016;15:772-774.

58. Menis D, Maroñas-Jiménez L, Delgado-Marquez AM, Postigo-Llorente C, Vanaclocha-Sebastián F. Two cases of severe hidradenitis suppurativa with failure of anakinra therapy. Br J Dermatol. 2015;172:810-811.

59. Zarchi K, Dufour DN, Jemec GB. Successful treatment of severe hidradenitis suppurativa with anakinra. JAMA Dermatol. 2013;149:1192-1194.

60. Leslie KS, Tripathi SV, Nguyen TV, Pauli M, Rosenblum MD. An openlabel study of anakinra for the treatment of moderate to severe hidradenitis suppurativa. JAm Acad Dermatol. 2014;70: 243-251.

61. Tzanetakou V, Kanni T, Giatrakou S, et al. Safety and efficacy of anakinra in severe hidradenitis suppurativa: a randomized clinical trial. JAMA Dermatol. 2016;152:52-59.
Clinical, Cosmetic and Investigational Dermatology

\section{Publish your work in this journal}

Clinical, Cosmetic and Investigational Dermatology is an international, peer-reviewed, open access, online journal that focuses on the latest clinical and experimental research in all aspects of skin disease and cosmetic interventions. This journal is included on PubMed. The manuscript management system is completely online
Dovepress

and includes a very quick and fair peer-review system, which is all easy to use. Visit http://www.dovepress.com/testimonials.php to read real quotes from published authors 